\title{
Effects of Different Fractions of Korean Red Ginseng on anti- diabetic effect in Type 2 Diabetic NSY Mice
}

* Min Jin Kim. Jung Hye Kim, Kahui Park, Ji Hong You, Sangbae Lee, Ji sun Nam, Jong Suk Park, Chul Woo Ahn, Kyung Rae Kim

\section{Objective and Aim}

Therapeutic properties of Korean red ginseng (KRG) on diabetes have been extensively introduced in the literature. Saponin, or ginsenoside, is considered as the major bioactive component mediating the therapeutic effects of KRG, the exact physiological mechanism underlying anti-diabetic effects is, however, still not fully elucidated. More than 30 different saponins together account for only about $3-4 \%$ of KRG, thereby, it is assumed that non-saponin fraction of KRG also carry potential anti-diabetic effects; however, there is no study reporting the differentiated effects of saponin and non-saponin fractions of KRG on glycemic indications and hyperglycemia-associated complication markers.

To clarify the differentiated effects of saponin and non-saponin fractions of KRG on glycemic indications and hyperglycemia-associated complication markers.

\section{Methods}

12-week-old male Nagoya-Shibata-Yasuda (NSY) mice were allocated into 4 groups: control group given standard rodent diet (SRD) or treatment groups given either Korean red ginseng extract (KRG), saponin fraction from KRG extract (Spn) or non-saponin fraction from KRG extract (NSpn) admixed in SRD. The targeted administration doses of KRG, Spn and NSpn were all 200 $\mathrm{mg} / \mathrm{kg} /$ day; all mice were fed assigned regimens for 24 weeks. Parameters for glycemic control, blood lipid profile, inflammation, oxidative stress, and antioxidant enzymatic activities were measured.

\section{Results}

Figure 1. Changes in fasting blood glucose

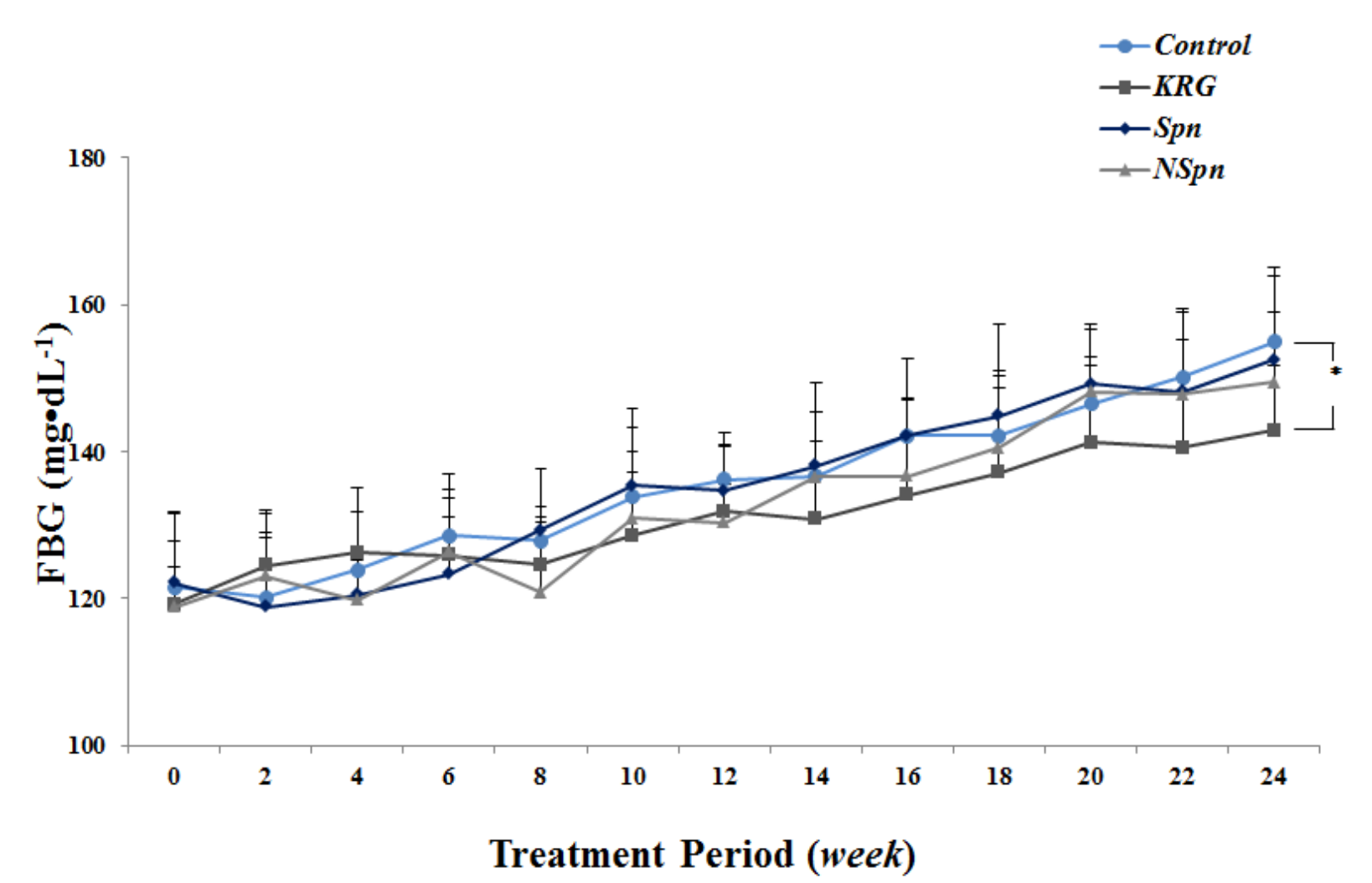

\section{Results}

Figure 2. Changes in parameters of glycemic control

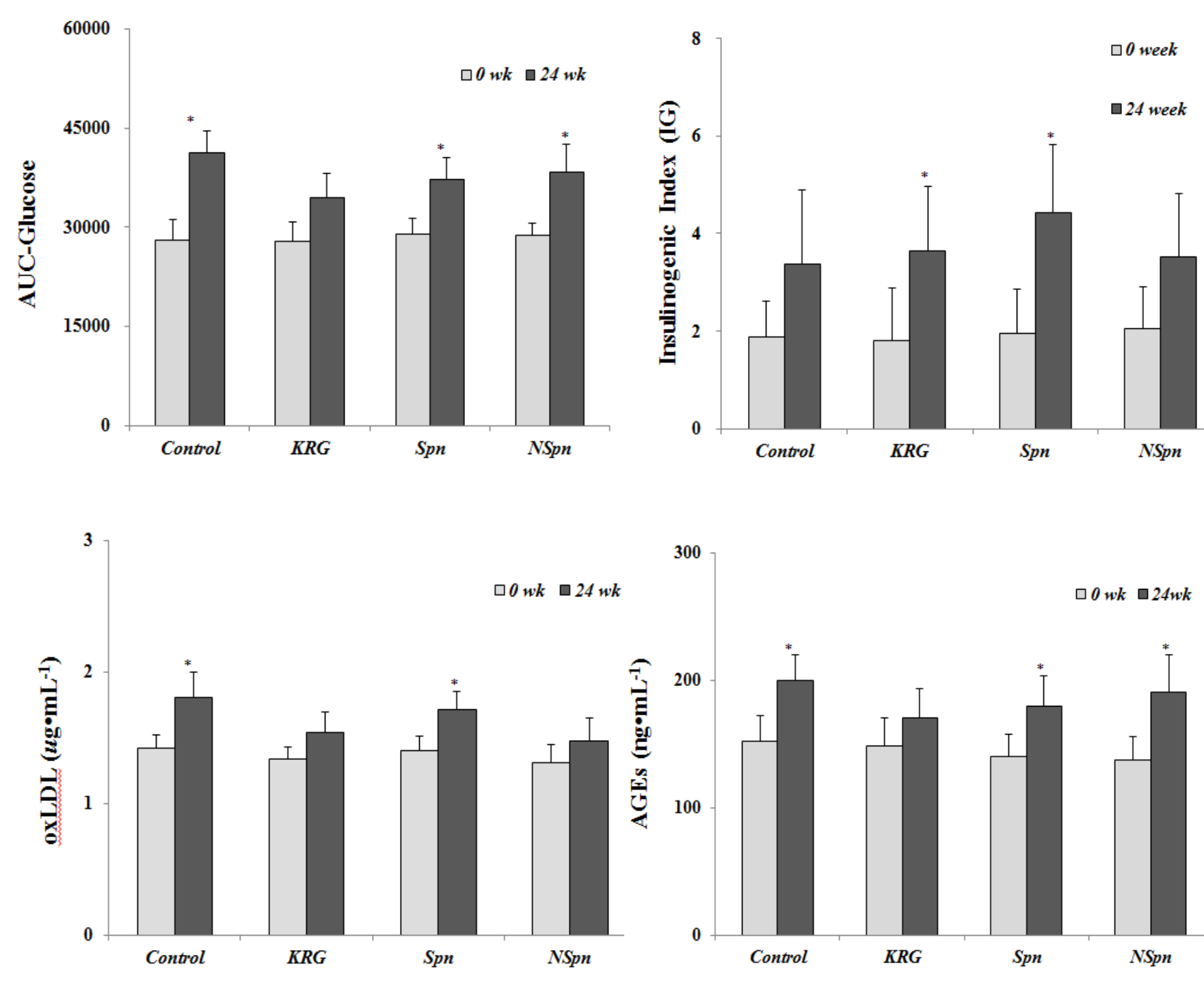

Abbreviation: end products

Figure 3. Accumulation of MDA(kind of AGEs) in skeletal muscle

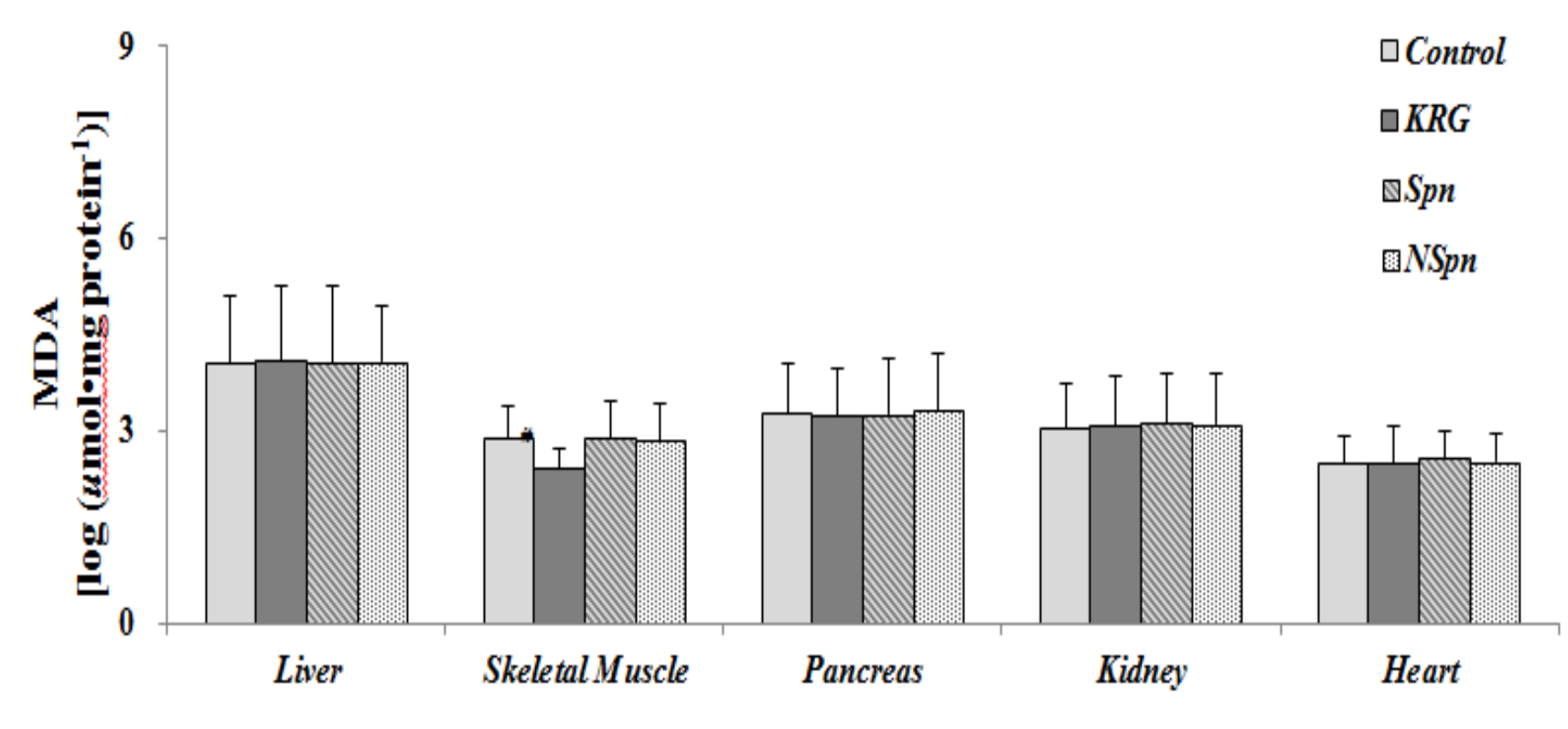

Abbreviation: MDA- Malonidialdehyde

\section{Conclusion}

This study showed that anti-diabetic properties of KRG are not mainly mediated by saponin, but the therapeutic potentials of KRG may be due to the orchestral effects of both saponin and non-saponin. 